\title{
Structural and Microstructural Characterization of Tin(II) Oxide Useful as Anode Material in Lithium Rechargeable Batteries Obtained from a Different Synthesis Route at Room Temperature
}

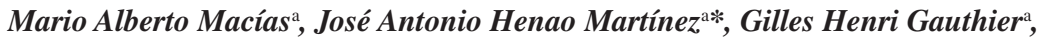 \\ Jorge Enrique Rodriguez $z^{\mathrm{b}}$,Humar Avila ${ }^{\mathrm{b}}$,José Pinto ${ }^{\mathrm{a}}$, Jesus Pinilla ${ }^{\mathrm{a}}$ \\ ${ }^{\mathrm{a}}$ Grupo de Investigación en Química Estructural - GIQUE, Escuela de Química, \\ Facultad de Ciencias, Universidad Industrial de Santander, A. A. 678, Bucaramanga, Colombia \\ ${ }^{\mathrm{b}}$ Grupo de Ciencia y Tecnología de Materiales Cerámicos - CYTEMAC, \\ Departamento de Física, Universidad del Cauca, Calle 5ta \# 4-70, \\ Popayán, Valle del Cauca, Colombia
}

Received: September 25, 2010; Revised: May 9, 2011

\begin{abstract}
Tin (II) oxide has been proposed as potential anode material in lithium rechargeable batteries. Different methods to obtain such compound have been developed with relative difficulty due to the fact that $\mathrm{Sn}$ (II) is easily oxidized to Sn(IV). We have applied a different methodology to synthesize SnO-romarchite by modifying the solvent nature of the controlled precipitation route using acetic acid and not water. Although the formation of $\mathrm{Sn}(\mathrm{IV})$ oxide could not be completely avoided, X-ray diffraction analysis confirmed the synthesis of metastable tin(II) oxide as major phase at room temperature. In depth analysis using Popa's model for Rietveld refinement allows to precise that the material corresponds to small and distorted crystallites, very anisotropic in size. SEM technique confirmed the microstructure is build of flower-like agglomerates of $\sim 15 \mu \mathrm{m}$, in turn made of plate-like individual grains that remind the crystallite structure anisotropy.
\end{abstract}

Keywords: romarchite, controlled precipitation method, microstructure, Rietveld method

\section{Introduction}

Among tin oxides, semiconducting $\mathrm{SnO}_{2}$ has been widely studied with many applications in photovoltaic cells, transparent electrodes, Li batteries, lasers or sensors ${ }^{1}$. Comparatively, tin(II) oxide $\mathrm{SnO}$ has been less studied but quite recently this material has received special attention due to interesting capacity values obtained as an anode material of lithium rechargeable batteries, especially when prepared in the form of amorphous tin-based composite ${ }^{1,2}$. During battery operation, it is possible to observe a first charging step that consists in a reaction between $\mathrm{Li}$ ions and $\mathrm{SnO}$, as shown in the following equation:

$$
6.4 e^{-}+6.4 \mathrm{Li}^{+}+\mathrm{SnO}_{(s)} \rightarrow \mathrm{Li}_{2} \mathrm{O}_{(s)}+\mathrm{Li}_{4.4} \mathrm{Sn}_{(s)}
$$

This step is followed by a reversible anode half-cell reaction in subsequent discharging-charging cycles, expressed as ${ }^{3}$ :

$$
\mathrm{Li}_{4.4} \mathrm{Sn}(s) \leftrightarrow 4.4 e^{-}+4.4 L i^{+}+\mathrm{Sn}_{(s)}
$$

The black-blue $\mathrm{SnO}$ powder synthesized for such an application always contains a small amount of $\mathrm{Sn}$ (IV) oxide due to traces of oxygen that promote $\mathrm{Sn}(\mathrm{II})$ oxidation and hinder the stabilization of the anode structure. Therefore, the main objective in $\mathrm{SnO}$ preparation is to minimize the oxidation of $\mathrm{Sn}(\mathrm{II})^{3-5}$. Moreover, the practical charge capacity is strongly dependent of the crystalline microstructure properties. Previous works have shown that a smaller crystallite size can facilitate the solid-state redox reactions, which produces higher practical reversible capacity ${ }^{3}$.
Different methods have been reported to synthesize pure and finely divided SnO powder, among them it is worth mentioning the following routes: homogeneous precipitation method using tin chloride and urea ${ }^{6}$, gas phase condensation process in inert gas atmosphere ${ }^{7}$, condensation/vaporization laser technique in diffusion chamber ${ }^{8}$ and solution process, in which amorphous oxo-hydroxo precursor of $\mathrm{Sn}^{2+}$ is crystallized via microwave heating ${ }^{9}$. Nevertheless, in all the above mentioned methods, $\mathrm{SnO}$ synthesis remains difficult and is currently accompanied by $\mathrm{SnO}_{2}$ formation ${ }^{10}$. Among the different ways of preparations, the solution synthesis technique is very attractive due to its ability to produce powdered materials with special metastable characteristics at ambient oxygen pressure, a requirement in the case of $\mathrm{SnO}$ preparation.

In the current work, we present an in depth analysis of both structural and microstructural characteristic of the Sn(II) oxide powder synthesized by controlled precipitation using acetic acid as a solvent instead of water, with the aim to describe the real state of the material and associate it in the future to its anodic properties in Li-battery application.

\section{Experimental Procedure}

The complete synthesis procedure was already described in a previous article ${ }^{11}$ and is briefly summarized here: $\mathrm{SnCl}_{2} \cdot 2 \mathrm{H}_{2} \mathrm{O}$ (Mallinckrodt, 98\%) was dissolved in glacial acetic acid (EM Science, $99.7 \%$ ) at $80{ }^{\circ} \mathrm{C}$. The $\mathrm{pH}$ was adjusted adding $\mathrm{NH}_{4} \mathrm{OH}$ to produce tin precursor precipitation. The suspension was then 
allowed to age for 24 hours at room temperature. A diethylamine $\left(\left(\mathrm{CH}_{3} \mathrm{CH}_{2}\right)_{2} \mathrm{NH}\right.$-Aldrich, $\left.98 \%\right)$ aqueous solution was finally poured to help removing chloride ions, before drying the as-obtained sample in an oven $\left(\mathrm{T}=80^{\circ} \mathrm{C}\right)$ for 24 hours.

A small portion of the obtained sample was gently ground in an agate mortar and sieved to a grain size less than $38 \mu \mathrm{m}$. The specimen was mounted on a zero-background specimen holder. During the sample preparation for acquisition of X-ray data, the possible tendency of the crystallites to align themselves parallel to the holder, as has been observed in other works ${ }^{9}$, was taken into account; in this way, the preferred orientation in the (002) direction was avoided by pouring the sample in a random way on the holder. X-ray powder diffraction (XRPD) data were collected at room temperature (RT) using a Rigaku D/MAX IIIB powder diffractometer working in Bragg-Brentano geometry and equipped with an $\mathrm{NaI}(\mathrm{Tl})$ scintillation detector. $\mathrm{A} \mathrm{Cu}$ $\mathrm{K} \alpha$ radiation $(\lambda=1.5406 \AA)$, a graphite monochromator, a fixed Soller slit, a divergence slit of $1^{\circ}$ and a $0.3 \mathrm{~mm}$ receiving slit was used. The diffractometer was operated over the range $2^{\circ}$ to $80^{\circ}(2 \theta)$ at $40 \mathrm{kV}$ and $35 \mathrm{~mA}$, using step scan with a step size of $0.02^{\circ}$ of $2 \theta$ and time of $6 \mathrm{~s} / \mathrm{step}$. The structural and microstructural analysis was carried out using the fitting software MAUD ${ }^{12}$ (Materials Analysis Using Diffraction), based on Rietveld method and Fourier analysis. For such refinements, a pseudo-Voigt peak-shape profile was used, adopting an iterative least-square procedure through minimization of the residual parameters $R_{w p}, R_{B}$ and $R_{\text {exp }}$. As will be described below, the crystallite size $(D)$ and the microstrain (root mean square, r.m.s. strain, $\left\langle\varepsilon^{2}\right\rangle^{1 / 2}$ ) were evaluated using the anisotropic Popa approach ${ }^{13}$. The microstructural analysis was realized by taking into account the instrumental contribution to the profile, for which the structural data of a $\mathrm{CeO}_{2}$ instrumental standard of known structural and microstructural characteristics were used ${ }^{14}$. The background variation was described by a five coefficients polynomial. Scanning Electron Microscopy was carried out using a JEOL $1200 \mathrm{EX}(80 \mathrm{KeV})$, mainly to determinate particle morphology and size.

\section{Results and Discussions}

An X-ray powder diffraction pattern can be well described by a convolution equation according to Enzo et al. ${ }^{15}$ :

$$
Y_{c}(2 \theta)=[B *(I * A)](2 \theta)+b k g
$$

where $Y c$ correspond to calculated intensity, $B$ is the sample broadening caused by microstructure, $I$ and $A$ are the symmetric and asymmetric instrumental contribution respectively, $b \mathrm{~kg}$ is the polynomial function of five degree for reproducing the background and $*$ correspond to convolution operation. In this sense, before the microstructural analysis by the Rietveld method, the instrumental contribution to the profile was calculated. Those refined parameters are summarized in Table 1 , where the $U, V$ and $W$ parameters considered in Caglioti equation for instrumental broadening ${ }^{16}$, besides instrumental asymmetry and Gaussianity of reflections are shown. Through the refinement, those parameters were fixed for estimation of sample contribution to the line profile shape.

A preliminary qualitative analysis was carried out to identify the presence of $\mathrm{SnO}$ in the obtained sample. The procedure confirmed that the obtained diffraction lines fit in a tetragonal symmetry with space group P4/nmm ( $\mathrm{N}^{\circ} 129$ ) according to the data for $\mathrm{SnO}$ (ICSD card No. 16481). The known structural information from database was used as starting point in the refinement procedure. As first step, the scale factor, background function and the unit cell parameters were refined in the first cycles of process. To carry out the structural refinement, the isotropic displacement factors (Biso) and the $z$ coordinate for $\mathrm{Sn}$ atoms were then refined.
For microstructural examination, the line profile analysis was carried out using a line broadening model with isotropic consideration which is described in Maud software as Delf, according to De Keijser et al. ${ }^{17}$. Table 2 shows the results of this preliminary analysis marked as $A$, where we can observe the poor fit caused by wrong values of structural parameters, especially in the atomic displacement factors.

Through a detailed observation of Figure 1, we finally observed a broad peak between $25-27^{\circ}$ in $2 \theta$ that was not well taken into account by the background function.

After database revision, the presence of $\mathrm{SnO}_{2}$ was confirmed as secondary phase (ICSD card No. 39178). The Rietveld refinement was then performed on the mixture and better results were obtained, reflected in the lower values of the residual parameters $R_{w p}, R_{B}$ and $R_{e x p}$, what is clearly observed graphically in Figure 2 . The refined parameters are shown in Table 2 , marked as $B$. Nevertheless, the $h k l$ dependence for peak broadening is evidenced in the inset of Figure 2.

Aiming to realize a deeper analysis, the refinement of $\mathrm{SnO}$ was then carried out using the size-strain-shape model developed by Popa $^{13}$.

In this model, $<R_{h k l}>$ was considered as the mean crystallite size in the crystallite direction $[h k l]$ developed in a convergent series of symmetrized spherical harmonics. In our case, the structure obtained belongs to the Laue group $4 / \mathrm{mmm}$, so $<R_{h k l}>$ is expressed as follows:

$$
<R_{h k l}>=R_{0}+R_{1} P_{2}^{0}(x)+R 2 P_{4}^{0}(x)+R 3 P_{4}^{4}(x) \cos 4 \varphi
$$

where $x=\cos \chi$. The terms $\chi$ and $\varphi$ correspond to co-latitude and azimuth angles respectively in the sample coordinate system ${ }^{18}$. The term $R_{0}$ corresponds to the mean crystallite size considering all the $[h k l]$ directions. During the microstructural analysis, a number of

Table 1. Instrumental parameters obtained from Rietveld analysis of a $\mathrm{CeO}_{2}$ sample with known structural and microstructural characteristics.

\begin{tabular}{cl}
\hline Instrumental parameter & Value obtained \\
\hline Zero-point position & $-0.00509(5)$ \\
Asymmetry coefficient 1 & $66.7(3)$ \\
Asymmetry coefficient 2 & $-0.059(1)$ \\
$U$ & $0.0048(1)$ \\
$V$ & $-0.00079(1)$ \\
$W$ & $0.0029(2)$ \\
Gaussianity coefficient 1 & $0.14(2)$ \\
Gaussianity coefficient 2 & $0.0058(3)$ \\
\hline
\end{tabular}

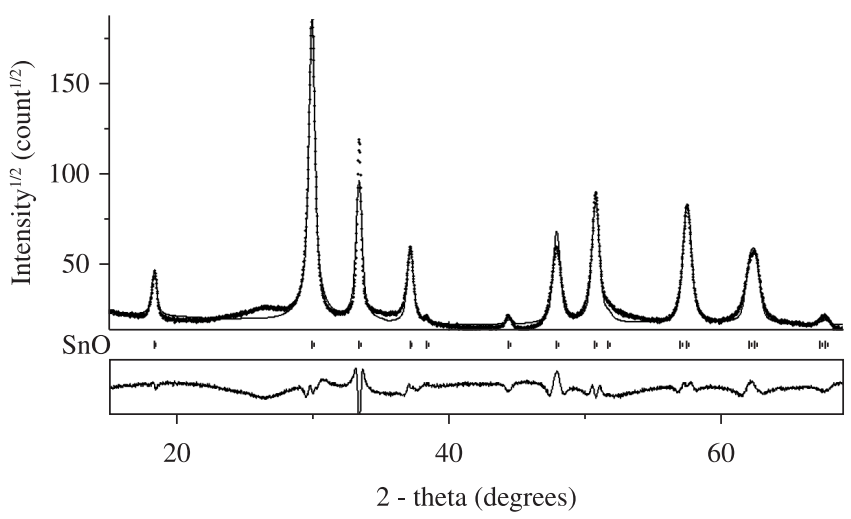

Figure 1. Identification of $\mathrm{SnO}$ phase. The refinement was realized using an isotropic line broadening. 
4 refinable parameters, $R_{n}$ with $n$ taking values from 0 to 3 , were determined by successive refinements starting from isotropic case, i.e. $\left\langle R_{h k l}\right\rangle=R_{0}$, and adding one by one until the corresponding difference in residuals values of refinement became insignificant. In fact, the number of these terms depends on the crystallite anisotropy ${ }^{18}$. Correspondingly, the microstrain was also determined for the corresponding Laue group:

$<\varepsilon_{h}^{2}>E_{h}^{4}=E_{1}\left(h^{4}+k^{4}\right)+E_{2} l^{4}+2 E_{3} h^{2} k^{2}+2 E_{4} l^{2}\left(k^{2}+h^{2}\right)$

This study revealed that the $\mathrm{SnO}$ compound shows anisotropic values for crystallite size and r.m.s. strain along the different $[h k l]$ directions. As is observed in Figure 3, corresponding to the best refinement result and marked as $C$ in Table 2, the peak broadening

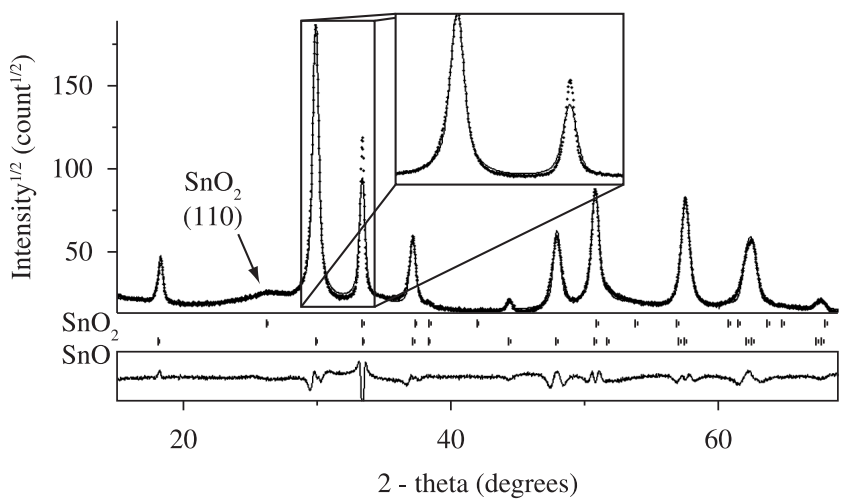

Figure 2. Identification of $\mathrm{SnO}_{2}$ as secondary phase. A broad peak is observed in the region of $2 \theta=25-27^{\circ}$ which correspond to the (110) reflection. In the inset is observed the $h k l$ dependence for peak broadening. of main phase clearly depends on crystallographic direction. Additionally, the corresponding difference between the observed and calculated profiles shows good fit, demonstrating that the proposed structural and microstructural models are correct.

On the other hand and due to the low contribution to the profile, the microstructural study of $\mathrm{SnO}_{2}$ phase was carried out using isotropic model of crystallite size and r.m.s. strain ${ }^{18}$. This analysis revealed strongly broadening of diffraction lines caused by the small size of the crystallites, $d=25(1) \mathrm{nm}$. This phase showed poor crystallization and the characteristics of a nanomaterial. The refinement of $\mathrm{SnO}_{2}$ took into account the crystallite size and cell parameters, maintaining fixed the atomic positions and isotropic displacement factors to the values observed in the ICSD card No. 39178.

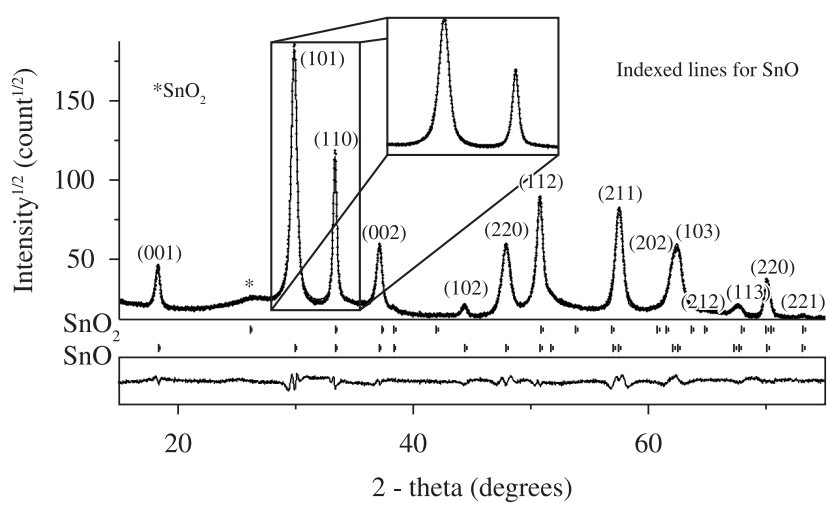

Figure 3. Graphical results of the final refinement of X-ray powder diffraction data for the obtained material adopting an anisotropic Popa model for $\mathrm{SnO}$ phase.

Table 2. Details obtained from Rietveld refinement analysis in each step, $A$ : Refinement of SnO phase with isotropic line broadening, $B$ : Refinement of mixture $\left(\mathrm{SnO}+\mathrm{SnO}_{2}\right)$ with isotropic line broadening, $C$ : Refinement of mixture $\left(\mathrm{SnO}+\mathrm{SnO}_{2}\right)$ with anisotropic line broadening for SnO. Only the data obtained for $\mathrm{SnO}$ are listed.

\begin{tabular}{|c|c|c|c|}
\hline & $A$ & $B$ & $C$ \\
\hline Crystal system & & Tetragonal & \\
\hline$a=b(\AA)$ & $3.8030(3)$ & $3.8026(2)$ & $3.8027(2)$ \\
\hline$c(\AA)$ & $4.8491(5)$ & $4.8479(4)$ & $4.8491(3)$ \\
\hline$V\left(\AA^{3}\right)$ & $70.13(4)$ & $70.53(4)$ & $70.12(3)$ \\
\hline $\mathrm{Z}$ & & 2 & \\
\hline Space group & & $P 4 / n m m\left(\mathrm{~N}^{\circ} 129\right)$ & \\
\hline $\mathrm{D}_{\text {calc }}\left(\mathrm{g} \cdot \mathrm{cm}^{-3}\right)$ & $6.377(4)$ & $6.341(4)$ & $6.378(3)$ \\
\hline $\mathrm{R}_{w p}$ & 16.86 & 14.58 & 8.52 \\
\hline $\mathrm{R}_{B}^{w p}$ & 12.20 & 10.24 & 6.72 \\
\hline $\mathrm{R}_{e x p}$ & 6.41 & 6.41 & 6.41 \\
\hline GoF & 2.56 & 2.27 & 1.33 \\
\hline Crystallite size parameters & $D=459(1) \AA$ & $D=428(3) \AA$ & $\begin{array}{c}R_{0}=641(4) \\
R_{1}=-789(19) \\
R_{2}=253(11) \\
R_{3}=101(18)\end{array}$ \\
\hline Microstrain parameters & r.m.s. $=0.00249(4)$ & r.m.s. $=0.00186(3)$ & $\begin{array}{c}E_{1}=0.00541(4) \\
E_{2}=0.00135(8) \\
E_{3}=-0.00503(6)\end{array}$ \\
\hline Refined structural parameters & $\begin{array}{c}\mathrm{B}_{\text {iso } \mathrm{Sn}}\left(\AA^{2}\right)=1.00(6) \\
\mathrm{B}_{\text {iso o }}\left(\AA^{2}\right)=2.9(4) \\
z_{\mathrm{Sn}}=0.2426(5)\end{array}$ & $\begin{array}{c}\mathrm{B}_{\text {iso } \mathrm{Sn}}\left(\AA^{2}\right)=0.82(5) \\
\mathrm{B}_{\text {iso o }}\left(\AA^{2}\right)=2.3(3) \\
z_{\mathrm{Sn}}=0.2407(4)\end{array}$ & $\begin{array}{c}\mathrm{B}_{\text {iso } \mathrm{Sn}}\left(\AA^{2}\right)=0.57(7) \\
\mathrm{B}_{\text {iso O }}\left(\AA^{2}\right)=0.99(9) \\
z_{\mathrm{Sn}}=0.2394(2)\end{array}$ \\
\hline
\end{tabular}

$\mathrm{B}_{\text {iso }}$ : Isotropic atomic displacement. $z$ : Refinable coordinate for $\mathrm{Sn}$ atom. During Rietveld analysis, in the case of $\mathrm{SnO}_{2}$, only the cell parameters were refined. The rest of the structural parameters were fixed to the values reported in ICSD card No. 39178. 
Table 3. Bond Valence Sum (BVS) calculations for Sn atoms in $\mathrm{SnO}$ phase.

\begin{tabular}{cc}
\hline & $\mathrm{Sn}$ \\
\hline$V i$ & 1.79 \\
Formal oxidation state & 2 \\
Rij tabulated parameter & $1.984(\AA)$ \\
$d i j$ observed bond length & $2.2277(\AA)$ \\
$b$ empirical constant & $0.37(\AA)$ \\
\hline
\end{tabular}

Best results (Table 2, case C) for complete structural parameters for $\mathrm{SnO}$ were obtained from this final part of refinement, which are in good agreement with published data. In particular, the axial ratio $c / a=1.27$ is in good agreement with the observed values previously reported ${ }^{19,20}$. The values of the Sn cation displacement parameters $\Delta \mathrm{z}=(1 / 4-z)$ and the internal distortion parameter $\sigma=\left[(1 / 4-x \mathrm{Sn})^{2}+(1 / 4-y \mathrm{Sn})^{2}+(1 / 4-z \mathrm{Sn})^{2}\right]^{1 / 2}$ are smaller compared to the previously values published by Pannetier and Denes ${ }^{21}$. The

Table 4. Microstructure parameters for $\mathrm{SnO}$ calculated by Maud software using the anisotropic Popa microstructural model: crystallite size $(D)$ and microstrain (r.m.s).

\begin{tabular}{cccccccc}
\hline $\mathrm{h}$ & $\mathrm{k}$ & $\mathrm{l}$ & Multiplicity & d-spacing & $(\mathrm{I} / \text { Io })_{\text {obs }}$ & Crystallite size D $(\mathrm{nm})$ & Microstrain $(\mathrm{r} . \mathrm{m} . \mathrm{s}).\left(10^{-4}\right)$ \\
\hline 0 & 0 & 1 & 2 & 4.8491 & 6 & $35.71(1)$ & $21.9(1)$ \\
1 & 0 & 1 & 8 & 2.9923 & 100 & $53.99(2)$ & $35.4(1)$ \\
1 & 1 & 0 & 4 & 2.6889 & 39 & $90.64(1)$ & $14.2(1)$ \\
0 & 0 & 2 & 2 & 2.4245 & 9 & $35.71(1)$ & $21.9(3)$ \\
1 & 1 & 1 & 8 & 2.3515 & 1 & $62.03(3)$ & $13.8(2)$ \\
1 & 0 & 2 & 8 & 2.0443 & 1 & $34.71(2)$ & $23.3(2)$ \\
2 & 0 & 0 & 4 & 1.9013 & 9 & $38.28(1)$ & $54.1(1)$ \\
1 & 1 & 2 & 8 & 1.8006 & 22 & $79.72(2)$ & $15.9(1)$ \\
2 & 1 & 1 & 16 & 1.6047 & 8 & $53.99(1)$ & $31.1(3)$ \\
2 & 0 & 2 & 8 & 1.4961 & 9 & $33.10(1)$ & $35.4(2)$ \\
1 & 0 & 3 & 8 & 1.4875 & 1 & $55.28(2)$ & $21.2(2)$ \\
2 & 1 & 2 & 16 & 1.3922 & 3 & $33.27(3)$ & $25.3(2)$ \\
1 & 1 & 3 & 8 & 1.3853 & 3 & $90.64(1)$ & $18.0(1)$ \\
2 & 2 & 0 & 4 & 1.3445 & $<1$ & & $14.2(3)$ \\
2 & 2 & 1 & 8 & 1.2955 & & & $13.9(1)$ \\
\hline
\end{tabular}

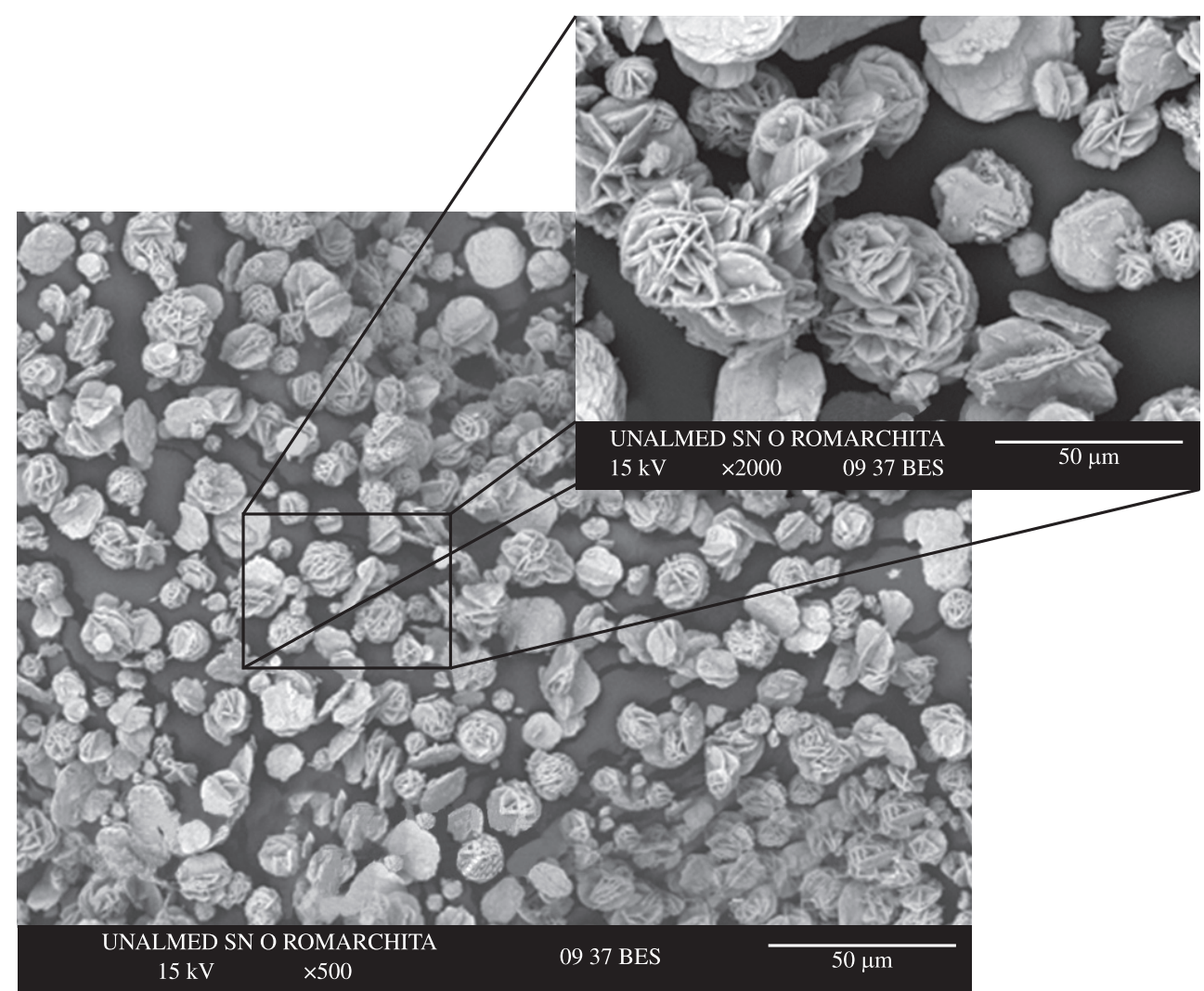

Figure 4. SEM images obtained from as-prepared $\mathrm{SnO}$. 
oxidation state of the corresponding Sn ion in the observed structure was calculated by analysis of the interatomic distance obtained from Rietveld analysis and using the Brown's Bond Valence (BVS) formula, in which the valence $V i$ of a atom is the sum of the individual bond valences $v_{i j}$ surrounding the atom:

$$
\sum_{j} v_{i j}=V_{i}
$$

The most commonly adopted empirical expression to extract the individual bond valences have been in turn calculated from the observed bond lengths using the following equation ${ }^{22}$ :

$$
v_{i j}=\exp \left[\frac{R_{i j}-d_{i j}}{b}\right]
$$

The results are shown in Table 3 and are in good agreement with the expected formal oxidation state of $\mathrm{Sn}^{2+}$.

A quantitative analysis was carried out using the Rietveld methodology. In the calculations was taken into account the following refinement equation:

$$
W_{i}=\frac{S_{i}(Z M V)_{i}}{\sum_{j} S_{j}(Z M V)_{j}}
$$

where $S$ is the refined scale factor, $Z$ the number of formula units per cell, $M$ is the molar mass in the unit formula and $V$ is the volume of the unit cell ${ }^{12,17}$.

Finally, after calculations, the sample reveals a composition of 80.6(4) wt. (\%) of $\mathrm{SnO}$ and 19.4(8) wt. (\%) of $\mathrm{SnO}_{2}$. This results shows that during crystallization process, oxidation of $\mathrm{Sn}$ (II) to $\mathrm{Sn}$ (IV) probably takes place, leading to the formation of $\mathrm{SnO}_{2}$ impurity.

The final microstructural results for $\mathrm{SnO}$ are shown in Table 4, where we can observe that the degree of anisotropy is pronounced, especially for Bragg direction (200), as a consequence of a crystallite shape (plate-like) reasonably far from spherical one. Despite anisotropic shape of crystallites, the mean value involving all different crystallographic directions is in good agreement with previous works, so its technological applications is not discarted ${ }^{3}$.

Figure 4 shows images obtained with SEM. Those micrographs show sand rose-shaped $\mathrm{SnO}$ agglomerates of about $15 \mu \mathrm{m}$ in size made of plate-shape grains whose thickness is well below the micron. Even if those grains are still an order of magnitude in size above the anisotropic dimensions of crystallites obtained from XRD analysis, the microstructural aspect of the sample is very well reflecting its structural and nanostructural characteristics. Similar results were reported previously by Pires et al. ${ }^{10}$ where the $\mathrm{SnO}$ powder was obtained by the microwave-assisted hydrothermal synthesis technique using $\mathrm{SnCl}_{2} \cdot 2 \mathrm{H}_{2} \mathrm{O}$ as a precursor and $\mathrm{NaOH}$ as mineralizing agent.

\section{Conclusion}

SnO-romarchite was synthesized by a new precipitation route in acetic acid. A certain degree of purity was obtained but, unfortunately, $\mathrm{SnO}_{2}$ formation was not completely avoided. X-ray diffraction analysis confirmed the formation of tin (II) oxide, metastable at room temperature, with tetragonal crystal system and space group $P 4 / \mathrm{nmm}$ $\left(\mathrm{N}^{\circ} 129\right)$, in agreement with literature data. The microstructural analysis revealed an anisotropic character of crystallites which could affect the material behavior in technological applications. This new alternative synthesis of tin oxide $(\mathrm{SnO})$ generates sand rose-shaped agglomerates of $\sim 15 \mu \mathrm{m}$, made of plate-like individual grains that remind the crystallite structure anisotropy.

\section{Acknowledgements}

The authors express their thanks to COLCIENCIAS Project Nr. 1103-14-17900 (Colombia) for financial support. Patricia Mosquera from the Microscopy Laboratory in the Universidad del Cauca, Popayán is greatly acknowledged as well as COLCIENCIAS for M.A. Macías's PhD scholarship.

\section{References}

1. Giefers H, Porsch F and Wortmann G. Kinetics of the disproportionation of SnO. Solid State Ionics. 2005; 176:199-207. http://dx.doi.org/10.1016/j. ssi.2004.06.006

2. Chouvin J, Branci C, Sarradin J, Olivier-Fourcade J, Jumas JC, Simon B et al. Lithium intercalation in tin oxide. Journal of Power Sources. 1999; 81:277-281. http://dx.doi.org/10.1016/S0378-7753(99)00140-8

3. Courtney IA and Dahn JR. Electrochemical and In-Situ X-ray Diffraction Studies of the Reaction of Lithium with Tin Oxide Composites. Journal of The Electrochemical Society.1997; 144:2045-2052. http://dx.doi. org/10.1149/1.1837740

4. Wu D-S, Han C-Y, Wang S-Y, Wu N-L and Rusakova IA. Microwave assisted solution synthesis of $\mathrm{SnO}$ nanocrystallites. Materials Letters.

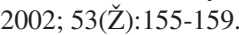

5. Giefers H, Porsch F and Wortmann G. Thermal disproportonation of SnO under high pressure. Solid state Ionics. 2005; 176:1327-1332. http://dx.doi.org/10.1016/j.ssi.2005.03.003

6. Kazumi F, Chizuko N, Keizo M and Shunmei M. Preparation of Tin(II) Oxide by a Homogeneous Precipitation Method. Bulletin of the Chemical Society of Japan. 1990; 63:2718-2720. http://dx.doi.org/10.1246/ bcsj.63.2718

7. Jimenez VM, Gonzalez-Elipe AR, Epinos JP, Justo A and Fernandez A. Synthesis of $\mathrm{SnO}$ and $\mathrm{SnO}_{2}$ nancrystalline powders by the gas phase condensation method. Sensors \& Actuators. 1996; 31(B):29.

8. Amitabh K and Rustum R. RESA- A wholly new process for fine oxide powder preparation. Journal Materials Research. 1988; 3:1373-1377. http://dx.doi.org/10.1557/JMR.1988.1373

9. Pires FI, Joanni E, Savu R, Zaghate MA, Longo E and Varela JA. Microwave-assisted hydrothermal synthesis of nanocrystalline SnO powders. Materials Letters. 2008; 62: 239-242. http://dx.doi. org/10.1016/j.matlet.2007.05.006

10. Pires FI, Joanni E, Savu R, Zaghete MA, Longo E and Varela JA. Microwave-assisted hydrothermal synthesis of nanocrystalline SnO powders. Materials Letters. 2008; 62:239-242. http://dx.doi. org/10.1016/j.matlet.2007.05.006

11. Avila HA and Rodríguez-Páez JE. Solvent effects in the synthesis process of tin oxide. Journal of Non-Crystalline Solids. 2009; 355:885-890. http://dx.doi.org/10.1016/j.jnoncrysol.2009.03.004

12. Lutterotti L. Material Analysis using Diffraction - MAUD: Computer code JAVA. Trento: University of Trento. 1997-2009. Available from: http://www.ing.unitn.it/ maud/

13. Popa NC. The (hkl) Dependence of Diffraction-Line Broadening Caused by Strain and Size for all Laue Groups in Rietveld Refine-ment. Journal of Applied Crystallography. 1998; 31:176-180. http://dx.doi.org/10.1107/ S0021889897009795

14. Balzar D, Audebrand N, Daymond MR, Fitch A, Hewat A, Langford $\mathrm{JI}$ et al. Size-strain line-broadening analysis of the ceria round-robin sample. Journal of Applied Crystallography. 2004; 37:911-924. http://dx.doi.org/10.1107/S0021889804022551

15. Enzo S, Fagherazzi G, Benedetti A and Polizzi S. A profile-fitting procedure for analysis of broadened X-ray diffraction peaks. I. Methodology. Journal of Applied Crystallography. 1988; 21:536-542. http://dx.doi.org/10.1107/S0021889888006612

16. Caglioti G, Paoletti A and Ricci FP. Nuclear Instrum. 1958; 3:223-228. http://dx.doi.org/10.1016/0369-643X(58)90029-X 
17. Young RA. editor. The Rietveld Method. Oxford: IUCr, Oxford University Press; 1993.

18. Chateigner D. Combined Analysis: structure-texture-microstructurephase-stresses-reflectivity determination by $X$-ray and neutron diffraction. Caen, France : CRISMAT-ENSICAEN. (UMR CNRS n ${ }^{\circ}$ 6508, 6 Bd. M. Juin, F-14050).

19. Izumi F. Pattern-fitting structure refinement of tin(II) oxide. Journal of Solid State Chemistry. 1981; 38(3):381-385. http://dx.doi.org/10.1016/00224596(81)90068-2
20. Pennetier J and Denes G. Tin(II) oxide: Structure refinement and thermal expansion. Acta Crystallographica B. 1980; 36:2763-2765. http://dx.doi. org/10.1107/S0567740880009934

21. Pannetier J and Denes G. Tin(II) oxide: structure refinement and thermal expansion. Acta Crystallographica B. 1980; 36:2763-2765. http://dx.doi. org/10.1107/S0567740880009934

22. Brese NE and O'Keeffe M. Bond-valence parameters for solids. Acta Crystallographica B. 1991; 47:192-197. http://dx.doi.org/10.1107/ S0108768190011041 